\title{
Customizable Tabular Access to Web Data Records for Convenient Low-vision Screen Magnifier Interaction
}

\author{
HAE-NA LEE, Stony Brook University \\ VIKAS ASHOK, Old Dominion University
}

\begin{abstract}
To interact with webpages, people with low vision typically rely on screen magnifier assistive technology that enlarges screen content and also enables them to pan the content to view the different portions of a webpage. This back-and-forth panning between different webpage portions makes it especially inconvenient and arduous for screen magnifier users to interact with web data records (e.g., list of flights, products, job advertisements), as this interaction typically involves making frequent comparisons between the data records based on their attributes, e.g., comparing available flights in a travel website based on their prices, durations. To address this issue, we present TableView+, an enhanced version of our previous TableView prototypea browser extension that leverages a state-of-the-art data extraction method to automatically identify and extract information in web data records, and subsequently presents the information to a screen magnifier user in a compactly arranged data table to facilitate easier comparisons between records. TableView + introduces new features aimed mostly at addressing the critical shortcomings of TableView, most notably the absence of interface customization options. In this regard, TableView+ enables low-vision users to customize the appearance of the data table based on their individual needs and eye conditions. TableView + also saves these customizations to automatically apply them to the best extent possible the next time the users interact with the data records on either the same or other similar websites. A user study with 25 low-vision participants showed that with TableView+, the panning time further decreased by $8.5 \%$ on unfamiliar websites and by $8.02 \%$ on a familiar website than with TableView when compared to a screen magnifier.
\end{abstract}

CCS Concepts: • Human-centered computing $\rightarrow$ Accessibility technologies; Empirical studies in accessibility;

Additional Key Words and Phrases: Web accessibility, usability, screen magnifier, low vision, visually impaired

\section{ACM Reference format:}

Hae-Na Lee and Vikas Ashok. 2022. Customizable Tabular Access to Web Data Records for Convenient Lowvision Screen Magnifier Interaction. ACM Trans. Access. Comput. 15, 2, Article 14 (May 2022), 22 pages.

https://doi.org/10.1145/3517044

\section{INTRODUCTION}

To interact with computer applications including web browsers, many people with low vision rely on screen magnifier assistive technology $[22,36,45]$ that enlarges screen content and also provides

This research work was funded by NSF awards 1805076, 1936027, 2113485, and NIH awards R01EY030085, R01HD097188. Authors' addresses: H.-N. Lee, Department of Computer Science, New Computer Science Bldg, Rm 230, Stony Brook University, Stony Brook, NY 11794; email: haenalee@cs.stonybrook.edu; V. Ashok, Department of Computer Science, E\&CS Bldg, Rm 3107, Old Dominion University, Norfolk, VA 23529; email: vganjigu@odu.edu.

Permission to make digital or hard copies of all or part of this work for personal or classroom use is granted without fee provided that copies are not made or distributed for profit or commercial advantage and that copies bear this notice and the full citation on the first page. Copyrights for components of this work owned by others than the author(s) must be honored. Abstracting with credit is permitted. To copy otherwise, or republish, to post on servers or to redistribute to lists, requires prior specific permission and/or a fee. Request permissions from permissions@acm.org.

(C) 2022 Copyright held by the owner/author(s). Publication rights licensed to ACM.

1936-7228/2022/05-ART14 \$15.00

https://doi.org/10.1145/3517044 


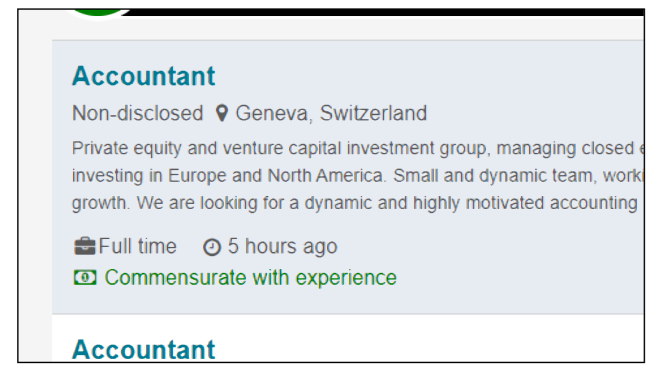

(a) Screen magnifier

\begin{tabular}{|l|l|l|l|}
\hline \multicolumn{1}{|c|}{ Title-link } & \multicolumn{1}{|c|}{ Save-icon } & Company-name & Location \\
\hline Accountant & Save & Non-disclosed & Geneva, Switze \\
\hline Accountant & Save & Carnegie Consul & London, UK \\
\hline Accountant & Save & Carnegie Consul & London, UK \\
\hline Accountant & Save & Walker Hamill & London, UK \\
\hline Accountant & Save & Hays Accountand & Melbourne VIC \\
\hline Accountant & Save & Redstone Comml New York. NY. \\
\hline
\end{tabular}

(b) Screen magnifier with TableView+

Fig. 1. Screenshots of a typical job-search website illustrating screen magnifier interaction with web data records using (a) screen magnifier with the zoom level set to $4 \times$; and (b) screen magnifier with TableView+, with the same zoom level of $4 \times$. Notice that with TableView+, information of more data records can be packed within the magnifier viewport. Furthermore, the width of any attribute column can be adjusted by simply dragging the border of that column in either direction with a pointing device.

other customization options such as color inversion, contrast enhancement, and cursor details. Enlarging a specific content pushes other portions off the screen, and therefore screen magnifier users have to pan (i.e., move the magnifier lens or viewport) application content with their pointing devices to access different portions of the content to complete their tasks. However, web tasks that involve navigating and comparing data records (i.e., search result items such as shopping products, available flights, and job listings) cannot be easily performed with just panning; the users also need to remember the details of the records as they pan the list of records, as only a small portion of this list is visible on screen at any time (see Figure 1(a)) when viewed under the magnifier lens. This additional cognitive burden often makes for an arduous and tedious interaction experience, especially when lists are long and the required enlargement (zoom level) is high [30]. While previous approaches $[7,8]$ can alleviate the panning and cognitive burden to a considerable extent using space-compaction techniques, they are not sufficient for facilitating easy and efficient interaction with web data records, as these records and their contents are typically stacked vertically in webpages (as in Figure 1(a)), and therefore just reducing the space is unlikely to push many occluded records within the magnifier lens or viewport. Hence, there is a need for an alternative interface that can facilitate quick and convenient comparison of data records based on their attributes (e.g., price, rating, description, shipping, number of reviews).

To fulfill this need, we present TableView+, a browser extension that automatically extracts data records from webpages using a state-of-the-art method [16] and then presents these records in a compact tabular form via a custom overlay popup interface. As illustrated in Figure 1(b), this compact tabular presentation of data is tailored for enabling easier and faster comparisons between data records, as more information can not only be packed within a smaller screen region, but also be shown close to each other within the magnifier viewport. TableView+ extends our prior TableView prototype [30] by supporting customization features that enable users to alter the appearance and organization of the data table (see Figure 1(b)) in real time depending on their individual needs. Specifically, the users can (i) adjust the column widths of individual attributes; (ii) filter the attributes to view only the data for preferred attributes; (iii) sort the data table records based on a preferred attribute; and (iv) increase and decrease the font size for more comfortable viewing of data. Furthermore, all these customizations are automatically saved by TableView + so they can be reused during subsequent accesses to either the same or similar websites, thereby mitigating the duplication of user effort. A user study with 25 low-vision screen magnifier users showed that 
TableView + further improved the user experience with web data records, compared to a state-ofthe-art space compaction method [8].

In sum, this work is an extension of our prior work that was published in ASSETS 2020 [30]. Our main contributions in this article are as follows:

- The design and development of TableView+, an extension of our previous prototype TableView, a browser extension that provides an alternative tabular presentation of web data records on a webpage. On top of the TableView features, TableView+ additionally supports interface personalization for enabling users to make (and also subsequently save/load) customizations to the data records table.

- The findings of a user study with 25 low-vision screen magnifier users evaluating the efficacy of TableView+ browser extension.

\section{RELATED LITERATURE}

Our work is closely related to existing literature on the following topics: (i) interaction behavior of low-vision users; (ii) improving usability for low-vision users; (iii) non-visual interaction with tabular data; and (iv) web data record extraction techniques.

\subsection{Interaction Needs and Behavior of People with Low Vision}

While there exists extensive literature aimed at understanding and addressing the usability needs of blind screen reader users $[6,7,9,19,40,41]$, the usability needs of low-vision screen magnifier users have been relatively underexplored [23, 31, 38, 48, 49]. Jacko et al. [23] focused on low-vision users who had age-related macular degeneration and analyzed the users' mouse cursor movements to observe the interaction strategies of the users. In their experiment, they found that the user performance was significantly impacted by the size of icons. Specifically, the performance improved as the icon size became larger. Szpiro et al. [48], however, investigated the general user behavior, interaction strategies, and challenges of screen magnifier users when they interacted with a variety of computing devices such as smartphones, tablets, and desktop computers. In their study, they observed that low-vision users typically used multiple assistive technologies simultaneously to meet interaction needs while interacting with computing applications on different devices. They also observed that users frequently needed to make multiple adjustments to comfortably view the application content.

The aforementioned studies focused only on generic aspects of low-vision interaction with computing devices and not specific aspects associated with browsing the web, especially interacting with data records. To the best of our knowledge, our prior work [30] is the first effort aimed at understanding the interaction challenges faced by low-vision screen magnifier users when they interact with web data records. In that work, we conducted an interview study with 16 low-vision screen magnifier users and subsequently uncovered many pain points related to interaction with web data records, most notably: (i) excessive panning necessitated by multiple revisits to the individual data records while making comparisons; (ii) undesired horizontal panning due to the spatially scattered arrangement of data record attributes (e.g., price, ratings, shipping details) with a lot of empty space between them; (iii) inability to distinguish between visited and unvisited data records; and (iv) inability to cover many data records due to fatigue and frustration. In sum, our interview study findings clearly indicated that an alternative screen magnifier-friendly presentation of data records was necessary to enhance the usability for low-vision users.

\subsection{Improving Usability for Low-vision Users}

Improving the user-interaction experience with GUIs for people with low vision has been previously explored [7, 8, 18, 28]. Specifically, Kline et al. [28] presented a set of accessibility tools that 
let users selectively magnify a portion of the screen area and also enabled them to keep track of mouse cursor location. Gajos et al. [18], however, proposed a personalized interface-generation technique for people with motor and low-vision impairments to interact with general computer applications. Their approach automatically generated personalized GUI from given interface specifications to match the custom needs of users based on their motor and vision abilities. Bigham et al. [7] developed a magnification system that automatically figured out how much to enlarge webpage content without introducing adverse side effects, such as additional horizontal scrolling. Similarly, Billah et al. [8] presented a context-preserving screen magnifier, named SteeringWheel, that attempted to keep semantically related web elements close to each other within magnified viewport, by discriminately magnifying only the non-whitespace content. Furthermore, they provided an easy-to-use Dial (a simple rotate-and-press input device) for low-vision users to interact with web content using simple gestures.

While the aforementioned solutions significantly improved usability, they were not tailored for the basic comparison tasks that screen magnifier users frequently perform almost on every website. For instance, the SteeringWheel system [8] enabled the screen magnifier users to sequentially navigate and view content of data records only one record at a time, and therefore it is not suitable for comparing multiple records. Also, the space-efficient magnification methods in both References [7] and [8] are mostly suitable for reducing the horizontal panning area of data records; these methods are less effective towards reducing the vertical panning area in data records and enabling easy comparisons, as there is generally less whitespace between the contents vertically (e.g., see Figure 1(a)). To facilitate easy comparisons between web data records, we previously designed TableView [30] that automatically extracted information in data records and then presented it as a compactly arranged table that was more amenable for screen magnifier interaction. In this work, we extend our prior TableView prototype by enabling screen magnifier users to customize the interface; TableView + automatically saves these customizations and then subsequently applies them to the interface the next time the users visit the same website or even other similar websites (e.g., those in the same domains such as shopping, travel, and job search).

\subsection{Non-visual Interaction with Tabular Data}

The accessibility and usability of tabular structures have been considerably explored in the prior research works [4, 5, 27, 47, 52]. However, almost all of these works have focused on blind screen reader users $[5,15,17,27,52]$. However, the research works on the usability of tables for low-vision screen magnifier users are sparingly few [38, 39]. In their study, Pascual et al. [39] found that all low-vision users could easily complete tasks while interacting with simple two-dimensional tables on an accessible website. Also, the average satisfaction rating for the tasks involving interaction with simple tables was very high (4 - Very easy). Their observations influenced our design choice for the data-record table in both the TableView interface [30] as well as the TableView+ interface in this article, where we used a two-dimensional table to display the information of data records.

\subsection{Web Data Record Extraction Techniques}

Plenty of extraction techniques have been previously devised to extract different kinds of data from webpages $[1,6,12,34,42]$, including data records $[3,53,55]$. For instance, the DOM treebased methods proposed in References [3, 16, 35, 51] focused on extracting data records, including the list of items such as search results, shopping products, and available flights, by exploiting repetitive patterns, such as the same XPaths, similar visual formatting, the same HTML subtree structure, tree alignment, and tree matching, in the webpage DOMs. However, Zhu et al. [55] proposed an integrated approach based on custom devised hierarchical conditional random fields for simultaneously detecting both data records and their attributes. As a preprocessing step, they 


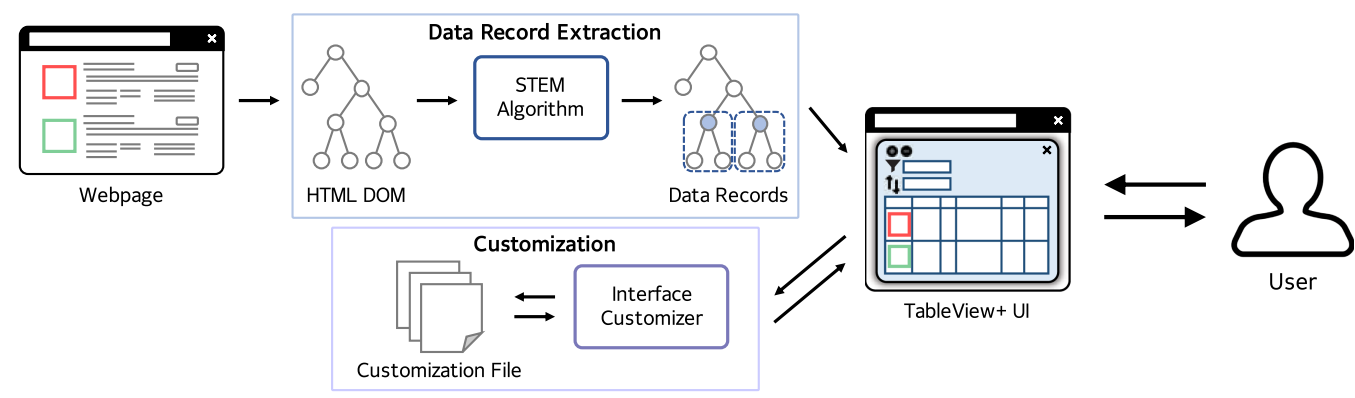

Fig. 2. TableView + architecture. TableView + UI containing a data table and customization options is shown as an overlay on top of the existing data records in the webpage. All user customization actions are recorded and stored locally. When applicable, these saved customizations are automatically applied before presenting the current data table.

relied on the VIPS webpage segmentation algorithm [13] to obtain the initial semantically meaningful blocks. Similarly, other visual segmentation-based approaches have also been suggested $[14,32,33]$, which exploit visual similarities to identify and extract data records. Given a plethora of existing algorithms for extracting data records, in this article, we leveraged a state-of-the-art algorithm, specifically the STEM algorithm [16], in TableView+.

\section{TABLEVIEW+ DESIGN}

Figure 2 presents an architectural workflow schematic of TableView + . When a webpage is loaded, TableView+ uses an existing accurate and robust data extraction technique, namely, the STEM algorithm [16], to automatically detect and capture information in web data records (if any) on the webpage. The extraction involves identifying relevant nodes in the HTML Document Object Model (DOM) of webpage that correspond to data records and their attributes (e.g., price, duration, and company on a flight reservation website). TableView+ also monitors the webpage for changes and re-extracts the data records from the webpage if the content is updated, e.g., when a user selects a set of search filters located adjacent to the data records in the webpage. The TableView+ extension also injects a button with the label TableView+ right above the data records. When the user clicks this button, the extracted data records and their attributes are displayed as a compact 2D data table in an interactive popup dialog, as shown previously in Figure 1(b). Hovering the mouse cursor over a table cell will generate an in-place overlay to display the entire contents of the cell (e.g., long descriptions). The data table allows the user to adjust the width of each column based on personal preferences. At the top of the interface, TableView+ provides buttons to increase or decrease font size, so the user does not have to switch focus to their screen magnifier's configuration dialog to adjust the font size while interacting with the TableView+ interface. The user can also selectively view only desired attributes of data records by leveraging attribute filters that are available above the data table in the popup dialog. There is Sort By option, too, just above the data table that lets the user sort the records in the table based on any desired attribute.

As shown in Figure 2, TableView + monitors and automatically saves all user customizations (i.e., column widths, attribute filters, sort choice, and font size) so they can be automatically applied to the best extent possible the next time the user interacts with data records on either the same website or similar websites. Note that it may not always be possible to automatically apply all the saved customizations, as the data records for a given item (e.g., electronics, apparel) in different websites or even different items on the same website may exhibit differences in their sets of attributes. Therefore, TableView+ only applies those saved customizations that are applicable 
to the current data table based on semantic similarities between the saved customization attribute names and the current webpage data record attribute names. We explain each of the TableView+ functionalities next.

\subsection{Data Record Extraction}

As mentioned earlier, we used the existing STEM (suffix tree-based extraction method) algorithm [16] due to its proven high precision of extraction as well as its robustness in filtering out noisy decorative elements in dense content-rich webpages. The core idea underlying the STEM algorithm is to find repetitive HTML tag path (similar to XPath) patterns in a webpage DOM. In this regard, the tag paths (e.g., <body $>,<u l>,<l i>$ ) of all the nodes in the DOM are computed, and then a unique integer is assigned to each unique tag path. Using these integer codes instead of the nodes, the entire webpage is then represented as a sequence of integers. From this webpage sequence, a suffix tree is constructed using the well-known suffix-tree construction algorithm [50]. This way, the task of finding the list of data records in a webpage is transformed into finding the corresponding repetitive node sequence in the constructed suffix tree. To find the correct node sequence, the STEM algorithm applies four custom data record specific filters to the candidate repetitive node sequences in the suffix tree, and then picks the most optimal node sequence as the final choice to extract the data records.

In Reference [16], the authors showed that the precision of the STEM algorithm on five different datasets were all above 0.9 , with the best precision being 0.986 . Similarly, they showed that the average recall, too, was very high-at least 0.8 for all five datasets, with the best recall being 0.961 . We also evaluated the STEM algorithm on our own custom dataset comprising 200 webpages (total of 6,397 data records) collected from the following categories: shopping, social media, entertainment, government, job search, booking, classifieds, and search engines. The choices for the webpages were based on the Alexa ranking [2] that keeps track of the top most-visited websites on the web. The collected 200 webpages containing data records were manually annotated by injecting custom data attributes in the webpage DOMs before saving them in the dataset. These injected data attributes serve as markers to measure the extraction performance of the STEM algorithm. The overall precision of identification on our custom dataset was 0.912 and the recall was 0.929 , thereby validating the capability of this algorithm in accurately identifying the data records. A closer inspection revealed that almost all errors were due to arbitrary advertisement content inside the data records, which made the underlying DOM subtrees of these records different from those of other data records on the webpage. Also, in some webpages, the advertisement content was arranged in the form of data records, thereby resulting in incorrect classification.

\subsection{TableView+ Interface}

The data table interface of TableView+ is shown in Figure 1(b). The motivation for choosing a tabular format to display data records in TableView + was influenced by findings of recent research studies [38, 52]. Specifically, Williams et al. [52] found that blind screen reader users could complete information lookup tasks much faster and with reduced cognitive effort when the entire webpages were transcoded as HTML tables. Also, Moreno et al. [38] observed that low-vision users preferred more coherent and organized content presentation, preferably at the center, so the movements in the field of vision that caused loss of context could be reduced.

Customization options. To further ease interaction with the data table as well as accommodate the important feature requests made by low-vision participants in a prior user study [30], TableView+ interface provides many options to customize the look of the data table. 


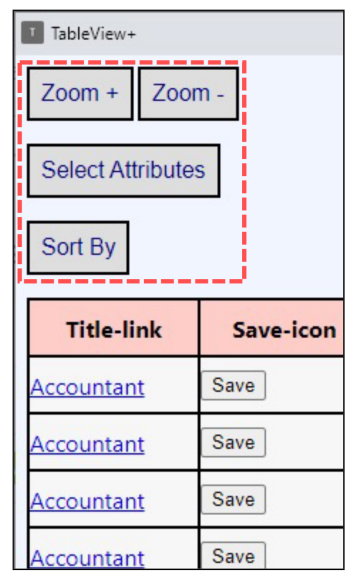

(a) Customization options

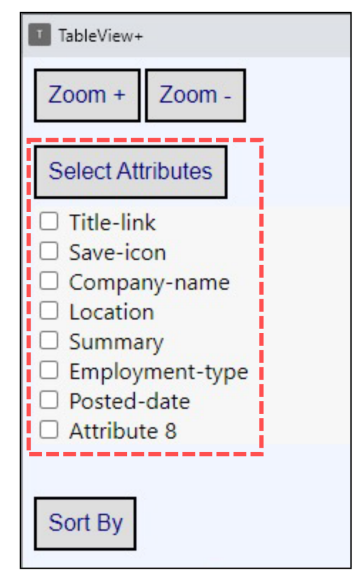

(b) Attribute selection

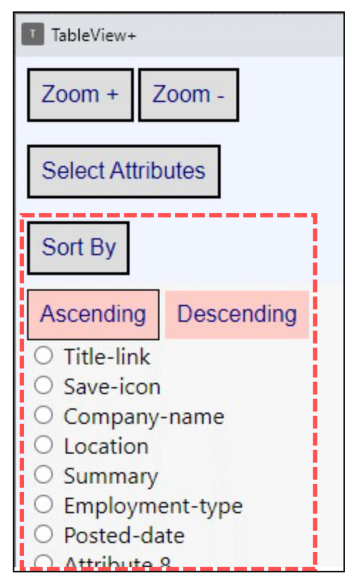

(c) Sorting option

Fig. 3. Customization options available in the TableView+ interface in addition to the option to modify the column widths of data table: (a) All options except column width adjustment are provided as buttons at the top of the interface. The column widths can be directly adjusted in the data table using a pointing device. Zoom+ and Zoom- buttons are included to change the font size of text in the interface; (b) Clicking the Select Attributes button displays the list of checkboxes from which a user can choose attributes to view in the data record table; and (c) Clicking the Sort By button displays a list of radio buttons that the user can choose from for sorting the data records.

First, TableView + allows a screen magnifier user to adjust the width of each column by simply placing a pointing device cursor on top of a column border and then dragging it either direction. Second, TableView+ enables the user to apply attribute filters for selectively viewing only the desired attributes of the data records in the data table. As shown in Figure 3(b), this feature is made available via the Select Attributes button at the top of the interface. When the user clicks on this button, a list of checkboxes with the corresponding attribute names, is made visible just below the Select Attributes button. The user can select the attributes (i.e., check the corresponding boxes) based on personal preferences, and the TableView+ accordingly updates the data record table in its interface to show only the selected columns. Note that by default TableView+ does not show this list of checkboxes to allocate more space for the actual data record table given the limited area of the magnifier viewport. Third, TableView + interface enables the user to sort the records in the data table based on any attribute. Similar to the Select Attributes functionality, the sort feature is made available via the Sort By button in the TableView+ interface, as shown in Figure 3(c). Clicking on the Sort By button displays a set of radio buttons with the corresponding attribute names just below the button, and the user can choose any one of the radio buttons to sort the data records based on the corresponding attribute. Ascending or descending options can also be chosen with "Ascending" or "Descending" buttons to decide the sorting order. As in case of Select Attributes, the sort radio buttons are hidden by default to assign more screen space to the data table. As mentioned earlier, the motivation underlying the inclusion of the aforementioned three customization features was based on the direct feedback provided by low-vision screen magnifier users in a prior study [30], where we evaluated the TableView prototype. Specifically, the participants explicitly expressed a desire for these customization options, as they felt that with these options, they could view more desired data record information within the magnifier viewport, while simultaneously reducing the amount of strenuous horizontal panning. 


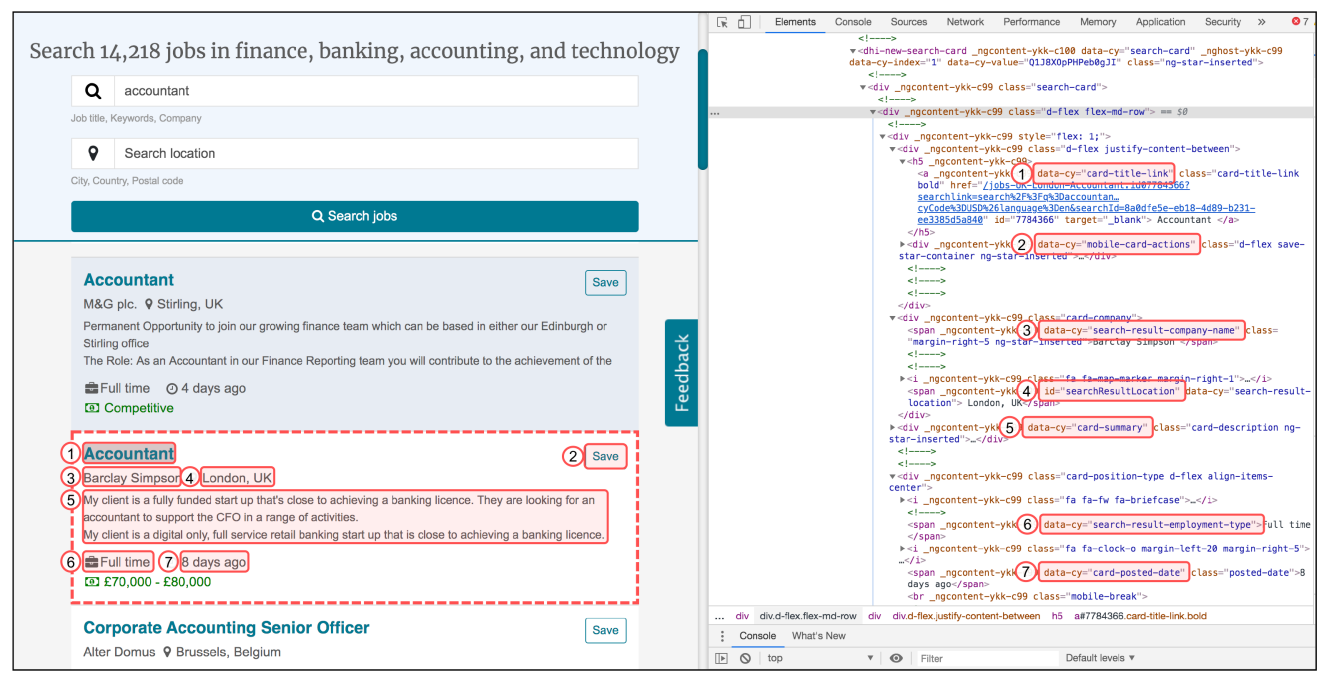

Fig. 4. An illustration of extracting attribute names from DOM metadata. As this example website has data attributes in the DOM, their values are best sources for extracting attribute names.

Apart from the Select Attributes and Sort By buttons, the TableView+ also provides Zoom+ and Zoom- buttons, as shown in Figure 3(a), to increase and decrease the font size of data table content, respectively. Based on our prior experience with low-vision screen magnifier users in usability studies $[8,29,30]$, these Zoom buttons come in handy for users who either (i) do not remember the browser's special shortcut for content enlargement or (ii) find it too tedious and frustrating to pan and shift focus to the screen magnifier's configuration window to do the same. The implementation details regarding the selection of attribute names, data table characteristics, and user-event handlers are described next.

Determining attribute names. TableView+ first tries to extract attribute names from the DOM metadata of the HTML nodes corresponding to the attributes of data record. Specifically, it checks if the attribute nodes or their descendants contain the "aria-label," "data," and "id" properties, and if so, it extracts them to be used as attribute names (see Figure 4). From the extracted metadata, we use a predefined dictionary of keywords to filter out the noisy textual content (e.g., "search," "search-result," and "card"). This dictionary was built by mining the commonly occurring noisy text in our aforementioned custom dataset. Also, 74\% (148) of the webpages in the dataset had one of the three properties ("aria-label," "data," "id") in the metadata of data-record attributes. In case this metadata is absent, TableView + simply generates a unique arbitrary name of the form "Attribute $i$," where $i$ is an integer.

Table characteristics. All attribute columns are initially assigned the same width, and the attribute columns are organized left-to-right in the same order as they appear in the DOM. However, the user can freely adjust the widths of individual columns based on custom needs and preferences. To view the full contents of an attribute for a particular record, the user simply needs to hover over the corresponding cell; an overlay will be dynamically generated to display the entire content of the cell. TableView+ automatically closes the overlay as soon as the user moves the cursor outside the cell boundaries. Also, based on the recommendations of Moreno et al. [38], TableView+ assigns the same font size to render the text in all table cells regardless of how they are actually rendered in the webpage. This default font size is selected to be the same as that of the first attribute 
of the data records in the webpage. Furthermore, to ensure consistency, TableView+ uses the same HTML element for rendering the attribute as that in the webpage containing the data records.

Relaying user actions. Some of the attributes of data records are actionable in the webpage (e.g., links, buttons), and therefore clicking on these attributes in the TableView+'s custom popup dialog interface should generate the same intended outcomes. Therefore, whenever the user clicks an actionable attribute (e.g., Save button), TableView + closes the popup dialog and simulates a click on the corresponding attribute in the original webpage, which often leads to loading of a new page in the browser. To facilitate this relay of user actions, TableView+ maintains a mapping between the table contents in its popup dialog with the corresponding attributes of data records in the webpage.

\subsection{Automatic Customization of Data Table Interface}

In our previous study [30], the participants explicitly mentioned that they would like to save their customizations so these customizations could be automatically applied the next time they visit the same or even similar webpages. However, providing support for automatic customization is not straightforward, as it involves many challenges. First, different websites may vary in their set of attributes (e.g., price, rating) shown for the same type of item (e.g., "laptop"). Second, even on the same website, attributes for different types of items (e.g., "television" vs. "t-shirt") are likely to differ with possibly a little overlap between them. Third, different websites may use different names or labels for the same attribute of an item (e.g., "rating" vs. "customer score").

To address the above challenges and thereby mitigate redundant manual effort from a user for each website that the user visits, TableView+ continuously monitors and automatically saves the following user customizations in a corresponding customization file so they can be automatically applied in subsequent accesses to the same or similar websites:

- Column Width: A list of <attribute name, column width> pairs capturing the dimensions of all attributes present in the data table.

- Undesired Attributes: A list of attribute names filtered out by the user using the Select Attributes option.

- Sort Attribute: The attribute name corresponding to the Sort By option if the user sorts the data table based on that attribute.

- Content Size: The font size of the table contents (if changed by the user with the Zoom+ or Zoom- options).

When the user visits a webpage containing data records, TableView+ executes the following routine to automatically apply the saved customizations to the current data table:

(1) If there exists a customization file for that website, go to step 3, else go to step 2.

(2) If there exists a customization file for a similar website, go to step 3, else stop and exit. Note, if there are multiple customization files, randomly pick one from the available pool.

(3) For each of the attribute names listed in the Undesired Attributes of the customization file, perform similarity checks with all the attribute names in the current data table; and if a match is found, remove that attribute (i.e., column) from the data table. Proceed to step 4.

(4) For each attribute name in the Column Width list of the customization file, perform similarity checks with all the attribute names in the current data table; and if a match is found, set the width of that attribute column to that specified in the Column Width list. Proceed to step 5.

(5) If Sort Attribute in the customization file is not empty, then sort the data table based on that attribute provided that the attribute is present in the current data table.

(6) If Content Size in the customization file is not empty, then change the font size of all text in the data table to that listed in the file. 
In step 2, to find similar websites, we employed the services of AlternativeTo ${ }^{1}$ crowdsourced platform. For this purpose, plenty of other alternatives (e.g., SimilarSites ${ }^{2}$ ) are also publicly available. In steps 3, 4, and 5 above, to determine if there exists a matching attribute in the current table for a given "query" attribute in the customization file, we leverage the well-known word2vec word embedding technique [37] available in the Gensim open-source library [43]. Specifically, for each attribute name in the data table, we compute the cosine similarity between its word2vec vector representation (computed by pretrained word2vec model) and the query attribute name's vector representation. If the highest similarity score generated after all these pairwise comparisons is greater than a predefined threshold $\tau=0.218$, then the corresponding attribute producing the highest score is considered a match to the query attribute.

To fix $\tau$ for successful matching, we built a custom dataset comprising 500 matching pairs of attribute names and 500 mismatching pairs of attribute names, where a match or mismatch was manually determined by a human annotator. Specifically, each pair was randomly selected from the attributes in actual data records (for a wide range of items such as clothing, jewelry, electronics, flights, hotel rooms, and jobs) on popular e-commerce websites. In this regard, we referred to the list of top 100 most-visited websites (Alexa ranking [2]) and selected English e-commerce websites from this list. With the cosine similarity score (CSS) between the word embeddings (obtained using pretrained word2vec model) of the attribute names in the pairs as a predictor variable, and the match ( 1 or 0$)$ as the outcome variable, we trained a logistic regression model that predicted the match probability $P$ (match) for a given CSS. We then computed the predicted probabilities for the CSS values from all the pairs in the dataset and sorted these probabilities in an increasing order In this sorted list, we noted the data point where $P($ match $)$ switched from less than 0.5 to greater than 0.5 , and the CSS value (0.218) corresponding to this point was selected as the threshold $\tau$ for separating successful and unsuccessful matches.

We opted for word2vec based approach as opposed to traditional dictionary based methods (e.g., WordNet) for similarity computation primarily because of its scalability and flexibility; dictionarybased methods are limited to a few recorded synonyms, whereas word representation-based methods have much wider coverage [20]. In fact, many prior works have also leveraged word2vec for word similarity based matching [24-26]. In sum, TableView+ inherits all the functionalities of TableView prototype [30], and additionally includes new features to address the key limitations of TableView that were uncovered in the prior user study. Table 1 presents a summary feature-wise comparison between TableView and TableView+.

\section{EVALUATION}

To determine if TableView+ further enhanced the user experience with web data records compared to the previous TableView [30] prototype, we conducted a user study with low-vision users. Specifically, our goal was to assess the extent to which TableView+ customization features such as column-width adjustment and automatic data-driven formatting further improved the interaction efficiency and usability for low-vision screen magnifier users. Moreover, through the study, we also intended to validate the prior results [30], given that there were concerns of external validity.

\subsection{Participants}

For the study, we recruited 25 low-vision participants, and their demographics are shown in Table 2 (Appendix A). To ensure external validity, we did not recruit people who had participated in our previous study where we evaluated TableView [30]. The inclusion criteria required

\footnotetext{
${ }^{1}$ https://alternativeto.net/.

${ }^{2}$ https://www.similarsites.com/.
} 
Table 1. Comparison between TableView and TableView+ with Regard to Supported Functionalities

\begin{tabular}{lll}
\hline \multicolumn{1}{c}{ Functionality } & \multicolumn{1}{c}{ TableView } & \multicolumn{1}{c}{ TableView+ } \\
\hline Data table presentation & Fixed size columns & Adjustable columns \\
\hline Attribute filtering & $\begin{array}{l}\text { "Filter" checkboxes to select } \\
\text { columns }\end{array}$ & $\begin{array}{l}\text { "Filter" checkboxes to select } \\
\text { columns }\end{array}$ \\
\hline Data record sorting & No support & $\begin{array}{l}\text { "Sort" radio buttons to order } \\
\text { based on any table column }\end{array}$ \\
\hline Increase/Decrease font & $\begin{array}{l}\text { Depend on screen magnifier } \\
\text { configuration dialog or } \\
\text { browser keyboard shortcut }\end{array}$ & $\begin{array}{l}\text { Explicit "Zoom" buttons to } \\
\text { change font size }\end{array}$ \\
\hline Save/Load customizations & No support & $\begin{array}{l}\text { All table customizations } \\
\text { automatically saved and } \\
\text { subsequently applied (to the } \\
\text { best extent possible) for the } \\
\text { same or similar websites }\end{array}$ \\
\hline
\end{tabular}

the participants to be regular screen magnifier users; low-vision users with extremely low visual acuity who relied on screen readers were excluded from the study. The average age of the participants was 48.16, (Median $=48$, Min $=29$, Max $=65)$. All participants were familiar with one of the two screen magnifiers, ZoomText [45] and Apple Zoom [22]; and they spent at least two hours a day browsing the web. Also, as seen in Table 2 (in Appendix A), the participant group was heterogeneous comprising a wide range of low-vision conditions based on diagnosis and visual acuity.

\subsection{Apparatus}

The study was conducted remotely, and the participants used their own computers to perform the study tasks. Zoom or Skype conferencing software was used for communication and screen sharing, and the entire session was recorded with the participants' permission. Eight participants (P3, P5, P6, P10, P14, P16, P19, and P23) used Zoom, Apple's built-in screen magnifier, on their MacBooks, and all other participants had ZoomText screen magnifier installed on their computers running Windows OS. All participants had Google Chrome installed on their computers. The TableView+ Chrome extension was shared (via Google Drive) with the participants. The experimenter assisted the participants in installing the extension via the conferencing software. Only five participants (P6, P10, P11, P12, and P15) required additional assistance from their caretakers or friends to install the browser extension.

\subsection{Design}

In a within-subject experiment, the participants were asked to perform representative "data-record interaction" tasks under the following study conditions:

- Screen Magnifier (SM): The participants only used their preferred screen magnifiers (e.g., ZoomText) to interact with data records specified in the tasks.

- Screen Magnifier \& Space Compaction (SC): The participants used their preferred screen magnifiers to interact with space-compacted data records. Space compaction is one of the state-of-the-art methods to improve low-vision interaction with web pages; for our study, we leveraged a recent space-compaction method proposed by Billah et al. [8] for this condition. 
- Screen Magnifier \& TableView (TV): The participants used their preferred screen magnifiers and TableView to interact with the data records.

- Screen Magnifier \& TableView + without preloaded customization (TVP): The participants used their preferred screen magnifiers and TableView+ to interact with data records. However, automatic customization of the data table was not done at the beginning based on saved customizations.

- Screen Magnifier \& TableView+ with preloaded customization (TVPC): The participants used their preferred screen magnifiers and TableView+ to interact with data records. Automatic customization of the data table was done at the beginning based on previously saved customizations (collected during the practice session in the study).

For convenience, the space-compaction algorithm and TableView were included into the TableView+ extension and could be turned "ON/OFF" with a checkbox selection in the extension GUI. For example, when the space compaction was turned "ON," TableView+ and TableView were automatically turned "OFF." In this way, we simulated the latter four study conditions using a single browser extension.

The study was divided into two parts, Parts A and B. In Part A, the participants did the tasks on unfamiliar websites, and in Part B, they did the tasks on a familiar website.

Part A - Unfamiliar websites. For each of the above conditions, the participants were asked to perform one task that required them to go over the first 15 data records in a website and select a record that satisfied pre-specified criteria comprising value constraints on the following job attributes: title, location, employment type, and company. To avoid learning effect, we selected five similar job search websites (https://www.efinancialcareers.com/, https:/www.snagajob.com/, https://www.roberthalf.com/, https://www.linkup.com/, and https://www.dice.com/) for the study. In each of these websites, data records (i.e., job listings) had regular repetitive structural patterns, and there were no advertisements interspersed within the list of records.

Furthermore, we vetted these websites to ensure that the STEM algorithm accurately extracted the data records to enable fair comparison between study conditions. All of the attributes in the pre-specified criteria were also vetted to ensure that all saved customizations concerning these attributes made during the practice session task on another similar website (https://www.usajobs gov/) could be successfully applied for all the five study websites. Furthermore, the search query for the data records in all these websites was fixed to be "accountant." During the recruitment phase, we confirmed that all participants were unfamiliar with these websites. The assignment of websites to conditions was randomized, and the ordering of the conditions was counterbalanced using the Latin square method [10]. We also ensured that the selection criteria was set to match the data record in the middle of the list in all of these websites. To avoid unforeseen issues, we used cached version of these webpages for the study.

Part B - Familiar website. For each of the above conditions, the participants were asked to perform a task that required them to go over the first 15 data records in a familiar website and select a record that satisfied pre-specified criteria on the following attributes: price, shipping, and company. Since all participants were familiar with the Amazon website, we chose that website for this part of the study. To avoid learning effect, five different queries ("laptop," "smartphone," "tv," "desktop," and "headphone") were used for each of the conditions. As in case of Part A, we set the selection criteria to match the data record in the middle of the list, and cached webpages were used for the study. The assignment of queries to conditions and the ordering of conditions were also counterbalanced using the Latin square method [10]. 


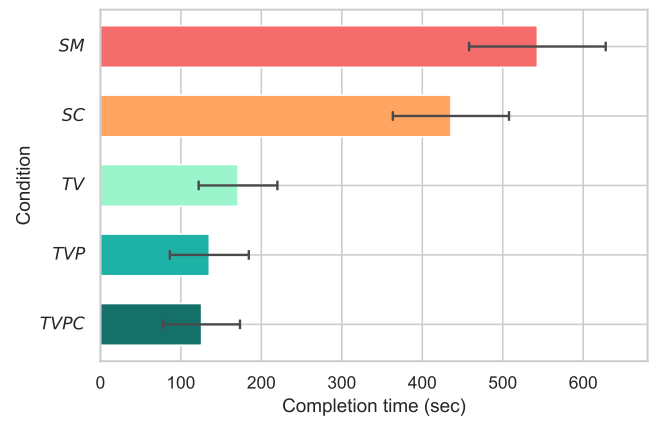

(a) Part A: Unfamiliar websites

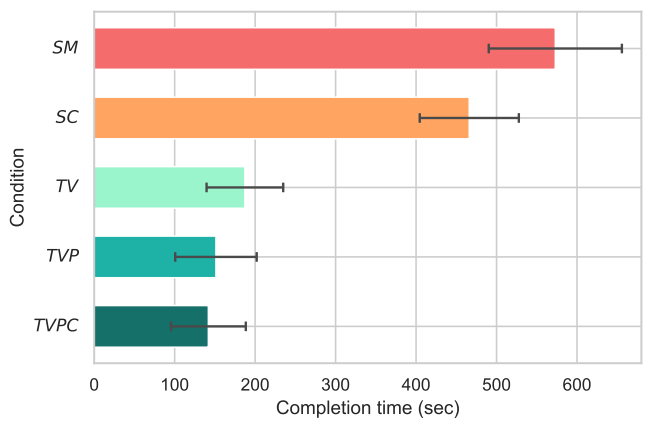

(b) Part B: Familiar website

Fig. 5. Average task completion times in different study conditions: Screen Magnifier (SM); Screen Magnifier \& Space Compaction (SC); Screen Magnifier \& TableView (TV); Screen Magnifier \& TableView+ without preloaded customization (TVP); and Screen Magnifier \& TableView+ with preloaded customization (TVPC).

\subsection{Procedure}

Before starting the experiment, the participants were given enough time to download and install the TableView+ extension either by themselves or with the assistance of their friends or family members. Then there was a practice session, where the participants familiarized themselves with study conditions and also did practice tasks. The practice tasks were similar to those in Parts A and B of the study except that we used different websites (Part A - https://www.usajobs.gov/ and Part B - https://www.walmart.com/) and different search queries ("programmer" and "microwave") to avoid any learning effect. The participants then completed the tasks in Part A of the study, followed by Part B. Since the task websites in Part A belonged to a different domain (job search) having different page layouts compared to those in Part B (shopping), there was no issue of learning effect, and therefore we did not counterbalance the tasks between the two parts of the study. The participants were assigned 15 minutes to complete each task. Post-study questionnaires were administered after each study condition. Exit interviews were conducted after the participants completed all their tasks. The screen-sharing and the recording features of the conference software were both turned on to ensure that all user-interaction activities were captured for later analysis. Each study lasted for three hours, and all conversations during the study were in English.

Measurements. We measured the completion times for each task during the study. Subjective measurements included the System Usability Scale (SUS) [11] and NASA Task Load Index (TLX) [21] scores, and qualitative subjective feedback were also collected. Errors, i.e., selecting incorrect data records, were recorded, and the participants were asked to continue the task and find the correct record, even after selecting incorrect records. Any peculiar interaction behavior during the study was also noted by the experimenter. To analyze the transcribed qualitative feedback, we used an open coding technique [44], where we iteratively went over the user responses and identified notable recurring themes in the data.

\subsection{Results}

Task completion times. Figures 5(a) and 5(b) present task completion time statistics for all five study conditions in Parts A and B of the study, respectively. Overall, there was a significant effect of the study conditions on the task completion times for both Part A (one-way ANOVA test, $F=230.85$, $p<0.001$ ) and Part B (one-way ANOVA test, $F=275.5, p<0.001$ ) of the study. As seen in Figures $5(\mathrm{a})$ and $5(\mathrm{~b})$, the TableView and TableView+ conditions performed significantly better than 
the baseline $S M$ and $S C$ conditions in both unfamiliar and familiar websites. Between TableView $(T V)$ and the two TableView+ conditions (TVP and TVPC), however, the TableView+ conditions (TVP and TVPC) performed slightly better than the TableView (TV) condition, in both unfamiliar and familiar websites. For Part A, post hoc pairwise comparisons using Tukey's HSD test between the following study conditions revealed significant differences: (a) $S M$ vs. $S C$ ( $Q=8.43, p<0.001)$; and (b) $S C$ vs. $T V(Q=20.76, p<0.001)$; however, we did not observe statistical significance between (c) $T V$ vs. $T V P(Q=2.79, p=0.282)$; and (d) TVP vs. $T V P C(Q=0.753, p=0.899)$. Similar observations were made for Part B: (a) $S M$ vs. $S C(Q=8.82, p<0.001)$; (b) $S C$ vs. $T V$ $(Q=23.00, p<0.001)$; (c) TV vs. TVP $(Q=2.96, p=0.227)$; and (d) TVP vs. TVPC $(Q=0.77$, $p=0.899)$. Notice that the task completion times were slightly higher for the familiar website (Part B) than for unfamiliar websites (Part A) for all conditions because the data records in the familiar website had more content than the records in the unfamiliar websites.

The results corresponding to the $S M, S C$, and $T V$ conditions were similar with those observed in our prior study [30]. Specifically, in that study, we observed that TableView reduced the task completion times by an average of $72.9 \%$ on unfamiliar websites (Part A) compared to those with just a screen magnifier (SM condition), and by $66.4 \%$ on the familiar website (Part B). In this study, the corresponding reductions with TableView were $68.6 \%$ and $67.3 \%$ on unfamiliar websites and the familiar website, respectively. With TableView+ (specifically, the TVPC condition), the task completion times were further reduced by $8.5 \%$ (avg.) for unfamiliar websites and by $8.02 \%$ for the familiar website. Also, in the prior study [30], the task completion times with TableView were $66.5 \%$ (avg.) lower than those in the other baseline SC condition for unfamiliar websites and $56.1 \%$ lower for the familiar website. In this study, with TableView, the corresponding reductions were $60.9 \%$ and $60.04 \%$ on unfamiliar websites and the familiar website, respectively. With TableView+, the reductions further increased by $10.5 \%$ for unfamiliar websites and $9.07 \%$ for the familiar website.

Analysis. In accordance with the findings of our prior study [30], we observed that in SM condition the participants could not view all the attributes of a data record in the magnifier viewport, and therefore they spent significant amount of time horizontally panning the content over large patches of whitespace to view all the attributes. This enlarged whitespace often caused disorientation, thereby leading the participants to unintentionally navigate to the neighboring data records without realizing this crossover. Realization and recovery from these accidental navigational mistakes further contributed to the completion time overhead. These accidental crossovers were slightly lower in case of the familiar website, as the participants were more cognizant of the website structure and data record layout. In the $S M$ condition, the participants also spent considerable time scrolling and panning vertically as very few data records were visible at any instant in the viewport due to content enlargement. The same was also observed in the $S C$ condition, since the space compaction did not contribute much in terms to reducing vertical whitespace, given that there was considerably less vertical space between the vertically stacked attributes within each data record. However, the horizontal panning and accidental crossovers were significantly reduced in the $S C$ condition, as space compaction was effective in shrinking the vast horizontal whitespace patches between data record attributes. These problems were not observed in the TableView and TableView+ conditions, as the data record details were reorganized and presented as a compact table in a separate overlay.

In the TableView $(T V)$ condition, the participants spent most of the time vertical scrolling over the data records. However, many participants (especially those using high magnification-greater than or equal to $5 \times$ zoom) spent some time horizontally panning over the table even after applying attribute filters, because the table could not completely fit within the viewport. TableView by default uses fixed column widths, and therefore some of the attributes that did not require the full 


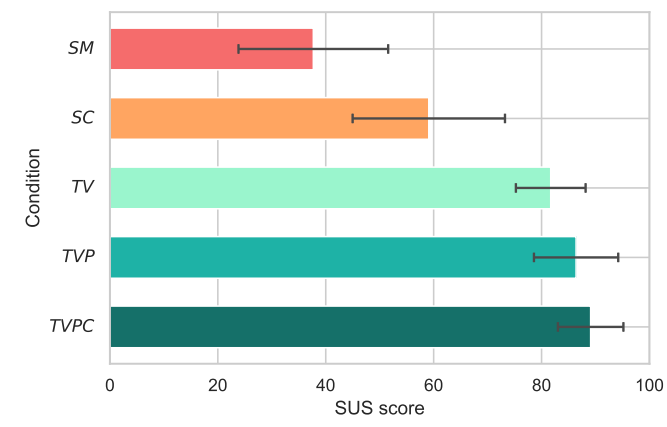

(a) SUS score

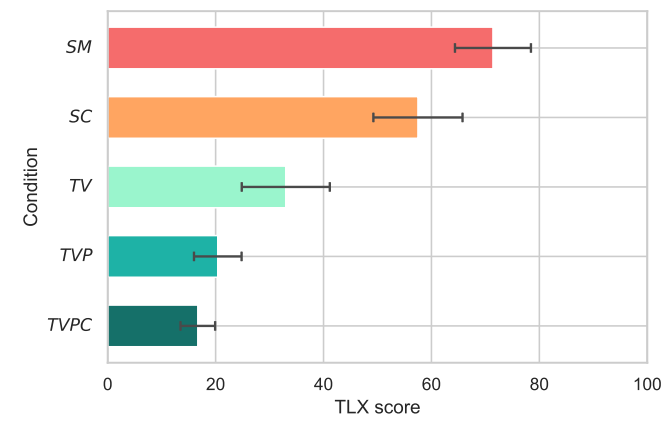

(b) NASA TLX score

Fig. 6. Average SUS and NASA TLX scores obtained from the participants under different study conditions: Screen Magnifier (SM); Screen Magnifier \& Space Compaction (SC); Screen Magnifier \& TableView (TV); Screen Magnifier \& TableView+ without preloaded customization (TVP); and Screen Magnifier \& TableView+ with preloaded customization (TVPC).

width were assigned more than necessary space in the viewport, which caused a small portion of the data table to go off screen, thereby necessitating horizontal panning.

However, in the TableView+ (TVP and TVPC) conditions, the participants cleverly adjusted the individual column widths as needed and thus were able to pack more content within the viewport; these customizations helped reduce the horizontal panning overhead. The slight increase in performance seen in the TVPC condition over the TVP condition was mainly due to the reduction in time spent on making these customizations, given that in the TVPC condition, many customizations including filters were automatically applied right at the beginning. Specifically, in the TVPC condition, the participants on average made $47.6 \%$ fewer customizations than in the TVP condition, while interacting with unfamiliar websites. For the familiar website, the reduction in the number of customization activities in the TVPC condition over the TVP condition was $56.4 \%$ (avg.). All of the customizations made in the TVPC condition were column-width adjustments (since attribute filters were automatically applied), which included instances where the participants overrode the automatic width customizations made by TableView + based on prior user data. These width readjustments (i.e., overrides) were done mostly because of the slight differences in contents of data records between different websites for the same items and also within the same website for different items. For the remaining automatic customizations such as filtering undesired attributes, sorting, and changing font size, the participants did not make any further adjustments, thereby demonstrating the effectiveness of TVPC condition.

Errors. Similar to the observations in our prior study [30], the total number of errors, i.e., selection of incorrect data record for a task, was the highest (44) for the baseline screen magnifier $(S M)$ condition compared to the other conditions $(S C=18, T V=8, T V P=5$, and $T V P C=4)$. This difference in the number of errors between conditions was found to be statistically significant (Kruskal-Wallis test, $H=23.59, p<0.001$ ).

The errors were predominantly due to the users forgetting the selection criteria, especially in the $S M$ condition. The participants attributed these errors to fatigue caused by excessive and tedious panning in the $S M$ condition. A small fraction (8.8\%) of these errors was also due to accidental crossovers to neighboring records panning over whitespace patches.

SUS and TLX scores. Figure 6 shows the statistics for the System Usability Scale (SUS) and NASA Task Load Index (TLX) scores for all the study conditions. Overall, there was a significant effect 
of the study conditions on the SUS scores (one-way ANOVA test, $F=108.86, p<0.001$ ). Pairwise comparisons between the following study conditions using the post hoc Tukey's HSD test also revealed significant differences: (a) $S M$ vs. $S C(Q=10.16, p<0.001)$; and (b) $S C$ vs. $T V(Q=10.73$, $p<0.001)$. However, we did not observe any statistically significant difference between (c) $T V$ vs. $\operatorname{TVP}(Q=2.23, p=0.511)$; and (d) TVP vs. TVPC $(Q=1.28, p=0.889)$. In sum, with TableView+ (particularly the TVPC condition), the SUS score increased by an additional 25.3\% (avg.) than with TableView when compared to the baseline $S M$ condition. Note that, since the participants had already formed their opinions about each condition during Part A of the study, we did not collect the SUS and TLX scores again for Part B of the study.

Regarding the interaction workload, as observable in Figure 6(b), the participants on average provided significantly more favorable (i.e., lower) ratings for the TableView $(T V)$ and TableView + (TVP and TVPC) conditions, compared to those for the $S M$ and $S C$ conditions. Specifically, we observed a significant effect of the study conditions on the TLX scores (one-way ANOVA test, $F=317.67, p<0.001)$. Statistically significant differences were also observed via the post hoc Tukey's HSD test between the following pairs of conditions: (a) $S M$ vs. $S C$ ( $Q=10.44, p<0.001$ ); (b) $S C$ vs. $T V(Q=18.27, p<0.001)$; and (c) $T V$ vs. $T V P(Q=9.48, p<0.001)$. However, we did not observe any statistical significant difference between TVP and TVPC $(Q=2.73$, $p=0.305)$. In sum, with TableView+, the TLX score (particularly the TVPC condition) decreased by an additional $23.3 \%$ (avg.) than with TableView when compared to the baseline $S M$ condition.

A closer inspection revealed that among the six sub-scales (i.e., Mental Demand, Physical Demand, Temporal Demand, Performance, Effort, and Frustration) of the two-part TLX questionnaire, "Effort" and "Frustration" sub-scales were the predominant discriminatory factors impacting the difference in TLX scores between study conditions-average 5.86 times and 5.84 times higher load ratings for the $S M$ condition compared to those for the TVPC condition, and average 4.45 times and 4.17 times higher load ratings for the $S C$ condition compared to those for the TVPC condition. These observations were slightly different from those in our prior study [30], where we found that "Mental Demand" and "Frustration" were the most discriminating factors driving the score differences between the $S M$ and $T V$ conditions. Interestingly, between the $T V$ and TVPC conditions, too, "Effort" (average 2.96 times higher load rating in the TV condition) and "Frustration" (average 2.1 times higher load rating in the $T V$ condition) were the dominant discriminating sub-scales contributing to the significant difference in the TLX scores.

Qualitative feedback. In the post-study interview, all participants stated that they were much more comfortable in the TableView and TableView + study conditions than in the SM and SC conditions. Moreover, they explicitly mentioned that the tabular arrangement of data records was much more amenable for making quick and easy comparisons than the conventional list or grid arrangement seen in the original websites. As in the prior study [30], a majority (18) of the participants expressed that horizontal panning (if needed) in the TableView and TableView+ interfaces was much less stressful than the status-quo horizontal panning in the websites, as there were no large patches of empty whitespace in the data table of TableView and TableView+. As explained by P12:

When I am directly interacting with the website, I have to pan in different directions to see different aspects of a product. There is a lot of empty space going from left to right, so it is hard to maintain direction when all you see on the screen is empty space. In the table, however, things are closer and also I can use the borders as markers to maintain my direction from left to right, and not accidentally slip to the next product. 
All participants with glaucoma (P5, P6, P12, P19, and P24) echoed their preferences for less panning and more scrolling as their counterparts in the earlier study [30]. To quote P19:

I have tunnel vision, so I need to pan more to see all the stuff on the screen. I would rather just look at one column and scroll, that way with a simple mouse action I can see all the data, and there is no need for me to go find stuff on my own.

However, almost all participants (except P4 and P18) mentioned that it was slightly frustrating to not be able to adjust the column widths for some of the attributes in the $T V$ condition-this was reflected in their responses to the TLX questionnaires discussed earlier. This was especially the case for all participants who did tasks in the TVP and TVPC conditions before doing the task in the $T V$ condition. These participants, on at least one occasion tried to adjust the column widths while doing the task in the $T V$ condition, before recalling that it was not possible to do so in that condition, which made some of them a little frustrated. Most (16) participants expressed that automatic application of previous customizations at the beginning of interaction (TVPC condition) was useful, but only to a certain extent. They rather wanted the TableView + system to be also able to cleverly adapt based on the size of content in the attributes of the data records in the current webpage. This was best expressed by the participant P20, a self-proclaimed expert computer user:

I liked that whatever I did before was reflected in the table of the next website, but I felt that much more could have be done to make it better. As I see it, I still needed to make customizations to interact comfortably. I do agree that some of the customizations I made cannot be predicted in advance, but some others such as decreasing a column size to reduce the unnecessary padding could have been done automatically. I am guessing this happened probably because the column in previous website had more content than the same column in the current website. Maybe, it is better for your system to remember what I intended to do rather than what I did.

All participants stated that the sorting and zooming options provided by TableView + were useful and necessary in general, though none of the participants used these options while doing any of the study tasks. When probed, they stated that the Select Attributes option was sufficient to do the study tasks. They also mentioned that when they were visually focused on the data table, they did not want to navigate all the way to the top of the interface to access these additional options. A few (4) participants even suggested that all customization options at the top should also be instantly accessible as overlays from anywhere in the data table.

\section{DISCUSSION}

The study results clearly demonstrated the improvement in overall performance achieved by TableView+ over TableView. However, the findings also illuminated new challenges and requirements that need to be addressed to further improve the low-vision user experience with web data records. We discuss some of these next.

Intelligent auto-customization of TableView+ data table appearance. As presented earlier, many participants (16) felt that the automatic customization feature of TableView+ could only partially accommodate their customization needs. This was mainly due to the TableView+ storing absolute column width values rather than the user intentions (e.g., reducing whitespace) underlying the column width adjustments. In this regard, a more sophisticated approach will be required to accommodate both the past user customizations and the content size/relative importance of attributes in the current webpage as influencing factors while determining the appropriate column widths for each of these attributes. Visual saliency $[46,54]$ might be a useful tool to measure relative importance of attributes. Mining patterns in past user behavior regarding attributes on webpages may 
also indicate their relative importance. We plan to explore all these options as part of our future work on this topic.

Identifying functional dependencies between page segments. In our prior work on the TableView prototype [30], we discovered that the participants also wanted support for other webpage segments (e.g., search filters, search form, sort options, previous/next page links) that directly improve interaction experience with data records. Specifically, they wanted these segments to be conveniently accessible while interacting with a data table in the TableView interface. While in TableView+, we did support the sort option, we have yet to include the other support segments. To support these additional segments, novel algorithms need to be designed that automatically identify and extract these support segments from the DOM of a webpage. Also, maintenance mechanisms should be devised to automatically fetch and refresh data records in the interface whenever search filters are applied.

Limitations and future work. A limitation of our TableView+ prototype is that like TableView, it currently works only on those websites where the third-party STEM algorithm is able to accurately extract data records. As mentioned earlier, this algorithm failed when there was high irregularity in structural patterns of data records, e.g., advertisements scattered between or within data records. Note that, in our study, we did not encounter this issue, because the webpages used for the tasks were all free of advertisements. Therefore, additional modifications to the original STEM algorithm and fail-safe mechanisms are needed to overcome these challenges. We also intend to explore semiautomatic user-assisted extraction algorithms, where the users can indicate the location of the data records by simply clicking on one of the records, and the algorithm residing in the browser extension can instantly extract all the records using the pattern matching techniques guided by users' assistance. All these are scope of our future research.

TableView+ also depends on an external word2vec embedding technique to determine the matches between attribute names belonging to different items in the same website or the same item in different websites. While this approach is well-known to be robust and accurate in many problem domains, it still depends on the availability of attribute names in the DOMs of webpages (see Section 3.2). It is possible that proper attribute names may not be specified in any of the "arialabel," "data," and "id" properties of the HTML nodes corresponding to the data record attributes. In such cases, the attribute names have to be extracted from other properties such as "class" that often have a lot of noisy extraneous text in addition to attribute names. These extraneous text can affect the performance of our word2vec-based attribute matching approach, and therefore more advanced preprocessing techniques are needed to filter out the noise before feeding the text to the word2vec model. This is also a part of our future work in improving TableView+. Another limitation of our TableView+ prototype was that some of the new customization options (Sort By and Zoom +/-) could not be conveniently accessed while interacting with the records in the data table. As a consequence, no participant used these features while doing the tasks during the study. To address this problem, we will explore alternative presentation techniques that can enable users to quickly access these features while simultaneously focusing on the content of data records.

\section{CONCLUSION}

This article presented TableView+, a browser extension that facilitates convenient interaction with web data records for low-vision screen magnifier users. TableView+, like its predecessor TableView, automatically identifies and extracts the information in data records on any webpage and then presents this information to the screen magnifier users in a compact data table via an interactive GUI. In addition, TableView+ lets users customize the appearance of the data table by simply dragging the borders of attribute columns in either direction to adjust their widths. TableView+ 
provides options for sorting and filtering records based on record attributes and also enables adjusting font size of data table content. All customizations are automatically saved by TableView+ so they can be reapplied to the best extent possible when the users revisit the same website or even other similar websites. A user study demonstrated the significantly higher efficacy of TableView + compared to TableView. Generalizing TableView + to automatically identify other segments that functionally depend on data records, and then display these segments in close proximity to the data table via an interactive interface, can further enhance the usability of TableView+.

\section{APPENDIX}

\section{A USER STUDY PARTICIPANT DEMOGRAPHICS}

Table 2. Participant Demographics

\begin{tabular}{|c|c|c|c|c|c|c|c|}
\hline \multirow{2}{*}{ ID } & \multirow{2}{*}{$\begin{array}{l}\text { Age/ } \\
\text { Gender }\end{array}$} & \multirow{2}{*}{ Diagnosis } & \multicolumn{2}{|c|}{ Visual Acuity } & \multirow{2}{*}{$\begin{array}{l}\text { Max } \\
\text { Zoom }\end{array}$} & \multirow{2}{*}{$\begin{array}{l}\text { Screen } \\
\text { Magnifier }\end{array}$} & \multirow{2}{*}{$\begin{array}{l}\text { Daily Web } \\
\text { Browsing }\end{array}$} \\
\hline & & & Left Eye & Right Eye & & & \\
\hline $\mathrm{P} 1$ & $35 / \mathrm{M}$ & Retinitis pigmentosa & $20 / 100$ & $20 / 200$ & $4 \times$ & ZoomText & 6 hours \\
\hline $\mathrm{P} 2$ & $58 / \mathrm{M}$ & Retinal detachment & $20 / 300$ & $20 / 300$ & $4 \times$ & ZoomText & 3 hours \\
\hline P3 & $47 / \mathrm{F}$ & Chorioretinal scarring & $20 / 100$ & $20 / 200$ & $4 \times$ & Zoom & 4 hours \\
\hline $\mathrm{P} 4$ & $42 / \mathrm{F}$ & Congenital retinal scar & $20 / 100$ & $20 / 200$ & $3 \times$ & ZoomText & 5 hours \\
\hline P5 & $60 / \mathrm{M}$ & Glaucoma & $20 / 500$ & $20 / 500$ & $6 \times$ & Zoom & 3 hours \\
\hline P6 & $56 / \mathrm{F}$ & Glaucoma & 0 & $20 / 400$ & $5 \times$ & Zoom & 3 hours \\
\hline P7 & $29 / \mathrm{M}$ & Stevens-Johnson syndrome & $20 / 400$ & $20 / 400$ & $5 \times$ & ZoomText & 5 hours \\
\hline P8 & $48 / \mathrm{M}$ & Cataracts & $20 / 100$ & $20 / 200$ & $4 \times$ & ZoomText & 4.5 hours \\
\hline P9 & $45 / \mathrm{M}$ & Optic atrophy & $20 / 500$ & $20 / 600$ & $5 \times$ & ZoomText & 4 hours \\
\hline P10 & $58 / \mathrm{F}$ & Cancer & $20 / 400$ & $20 / 400$ & $6 \times$ & Zoom & 4 hours \\
\hline P11 & $65 / \mathrm{F}$ & Diabetic retinopathy & $20 / 600$ & $20 / 500$ & $6 \times$ & ZoomText & 2 hours \\
\hline $\mathrm{P} 12$ & $63 / \mathrm{F}$ & Glaucoma & $20 / 100$ & 0 & $4 \times$ & ZoomText & 3.5 hours \\
\hline P13 & $45 / \mathrm{M}$ & Chorioretinal scarring & $20 / 500$ & $20 / 500$ & $6 \times$ & ZoomText & 4 hours \\
\hline P14 & $49 / \mathrm{M}$ & Diabetic retinopathy & $20 / 400$ & $20 / 200$ & $5 \times$ & Zoom & 5 hours \\
\hline $\mathrm{P} 15$ & $53 / \mathrm{M}$ & Optic atrophy & 0 & $20 / 400$ & $5 \times$ & ZoomText & 5 hours \\
\hline P16 & $48 / \mathrm{F}$ & Congenital retinal scar & 0 & $20 / 600$ & $7 \times$ & Zoom & 4 hours \\
\hline P17 & $34 / \mathrm{M}$ & Nystagmus & $20 / 200$ & $20 / 400$ & $5 \times$ & ZoomText & 5 hours \\
\hline P18 & $45 / \mathrm{F}$ & Stargardt disease & $20 / 200$ & $20 / 100$ & $3 \times$ & ZoomText & 5 hours \\
\hline P19 & $41 / \mathrm{M}$ & Glaucoma & $20 / 400$ & $20 / 300$ & $5 \times$ & Zoom & 5 hours \\
\hline $\mathrm{P} 20$ & $43 / \mathrm{M}$ & Optic atrophy & $20 / 200$ & $20 / 200$ & $4 \times$ & ZoomText & 4 hours \\
\hline $\mathrm{P} 21$ & $50 / \mathrm{F}$ & Astigmatism & $20 / 200$ & $20 / 400$ & $5 \times$ & ZoomText & 4.5 hours \\
\hline $\mathrm{P} 22$ & $41 / \mathrm{F}$ & Retinitis pigmentosa & 0 & $20 / 600$ & $7 \times$ & ZoomText & 4 hours \\
\hline $\mathrm{P} 23$ & $57 / \mathrm{M}$ & Diabetic retinopathy & $20 / 400$ & 0 & $4 \times$ & Zoom & 3 hours \\
\hline $\mathrm{P} 24$ & $43 / \mathrm{M}$ & Glaucoma & $20 / 300$ & $20 / 200$ & $5 \times$ & ZoomText & 5 hours \\
\hline $\mathrm{P} 25$ & $49 / \mathrm{F}$ & Leber congenital amaurosis & $20 / 600$ & 0 & $8 \times$ & ZoomText & 5 hours \\
\hline
\end{tabular}

All data was self-reported. The Screen Magnifier column refers to the participants' preferred screen magnifiers-the participants used these magnifiers to perform the tasks in the user study.

\section{ACKNOWLEDGMENTS}

We thank anonymous reviewers for their insightful feedback.

\section{REFERENCES}

[1] Julian Alarte, David Insa, and Josep Silva. 2017. Webpage menu detection based on DOM. In SOFSEM 2017: Theory and Practice of Computer Science, Bernhard Steffen, Christel Baier, Mark van den Brand, Johann Eder, Mike Hinchey, and Tiziana Margaria (Eds.). Springer International Publishing, Cham, 411-422.

[2] Alexa Internet, Inc. 2021. Alexa - Top sites. Retrieved from https://www.alexa.com/topsites. 
[3] Manuel Álvarez, Alberto Pan, Juan Raposo, Fernando Bellas, and Fidel Cacheda. 2007. Finding and extracting data records from web pages. In Proceedings of the International Conference on Embedded and Ubiquitous Computing (EUC'07). Springer-Verlag, Berlin, 466-478.

[4] Dagmar Amtmann, Kurt Johnson, and Debbie Cook. 2002. Making web-based tables accessible for users of screen readers. Libr. Hi Tech 20, 2 (2002), 221. Retrieved from https://www.learntechlib.org/p/96485.

[5] Chieko Asakawa and Takashi Itoh. 1999. User interface of a nonvisual table navigation method. In CHI'99 Extended Abstracts on Human Factors in Computing Systems (CHI EA'99). Association for Computing Machinery, New York, NY, 214-215. DOI : https://doi.org/10.1145/632716.632850

[6] Vikas Ashok, Yury Puzis, Yevgen Borodin, and I. V. Ramakrishnan. 2017. Web screen reading automation assistance using semantic abstraction. In Proceedings of the 22nd International Conference on Intelligent User Interfaces (IUI'17) Association for Computing Machinery, New York, NY, 407-418. DOI : https://doi.org/10.1145/3025171.3025229

[7] Jeffrey P. Bigham. 2014. Making the web easier to see with opportunistic accessibility improvement. In Proceedings of the 27th Annual ACM Symposium on User Interface Software and Technology (UIST'14). ACM, New York, NY, 117-122. DOI : https://doi.org/10.1145/2642918.2647357

[8] Syed Masum Billah, Vikas Ashok, Donald E. Porter, and I. V. Ramakrishnan. 2018. SteeringWheel: A locality-preserving magnification interface for low vision web browsing. In Proceedings of the CHI Conference on Human Factors in Computing Systems (CHI'18). ACM, New York, NY. DOI : https://doi.org/10.1145/3173574.3173594

[9] Yevgen Borodin, Jeffrey P. Bigham, Glenn Dausch, and I. V. Ramakrishnan. 2010. More than meets the eye: A survey of screen-reader browsing strategies. In Proceedings of the International Cross Disciplinary Conference on Web Accessibility (W4A'10). ACM, New York, NY. DOI : https://doi.org/10.1145/1805986.1806005

[10] James V. Bradley. 1958. Complete counterbalancing of immediate sequential effects in a Latin square design. J. Amer. Statist. Assoc. 53, 282 (1958), 525-528.

[11] John Brooke et al. 1996. SUS-A quick and dirty usability scale. Usab. Eval. Industr. 189, 194 (1996), 4-7.

[12] Deng Cai, Shipeng Yu, Ji-Rong Wen, and Wei-Ying Ma. 2003. VIPS: A Vision-based Page Segmentation Algorithm. Technical Report MSR-TR-2003-79. Retrieved from https://www.microsoft.com/en-us/research/publication/vips-a-visionbased-page-segmentation-algorithm/.

[13] Deng Cai, Shipeng Yu, Ji-Rong Wen, and Wei-Ying Ma. 2004. Block-based web search. In Proceedings of the 27th Annual International ACM SIGIR Conference on Research and Development in Information Retrieval (SIGIR'04). Association for Computing Machinery, New York, NY, 456-463. DOI : https://doi.org/10.1145/1008992.1009070

[14] Zehuan Cai, Jin Liu, Lamei Xu, Chunyong Yin, and Jin Wang. 2017. A vision recognition based method for web data extraction. Adv. Sci. Technol. Lett. 143 (2017), 193-198.

[15] Mechmet Chiousemoglou and Helmut Jürgensen. 2011. Setting the table for the blind. In Proceedings of the 4th International Conference on PErvasive Technologies Related to Assistive Environments (PETRA'11). Association for Computing Machinery, New York, NY. DOI : https://doi.org/10.1145/2141622.2141624

[16] Yixiang Fang, Xiaoqin Xie, Xiaofeng Zhang, Reynold Cheng, and Zhiqiang Zhang. 2018. STEM: A suffix tree-based method for web data records extraction. Knowl. Inf. Syst. 55, 2 (2018), 305-331.

[17] António Ramires Fernandes, Alexandre Carvalho, José João Almeida, and Alberto Simoes. 2006. Transcoding for web accessibility for the blind: Semantics from structure. In Digital Spectrum: Integrating Technology and Culture - Proceedings of the 10th International Conference on Electronic Publishing held in Bansko, Bulgaria 14-16 June 2006, Bob Martens and Milena Dobreva (Eds.). 123-134. https://www.researchgate.net/publication/221436068_Transcoding_for_Web_ Accessibility_for_the_Blind_Semantics_from_Structure.

[18] Krzysztof Z. Gajos, Jacob O. Wobbrock, and Daniel S. Weld. 2007. Automatically generating user interfaces adapted to users' motor and vision capabilities. In Proceedings of the 20th Annual ACM Symposium on User Interface Software and Technology) (UIST'07). ACM, New York, NY, 231-240. DOI : https://doi.org/10.1145/1294211.1294253

[19] Steven Gardiner, Anthony Tomasic, and John Zimmerman. 2015. EnTable: Rewriting web data sets as accessible tables. In Proceedings of the 17th International ACM SIGACCESS Conference on Computers \& Accessibility (ASSETS'15). Association for Computing Machinery, New York, NY, 443-444. DOI : https://doi.org/10.1145/2700648.2811344

[20] Abram Handler. 2014. An empirical study of semantic similarity in WordNet and Word2Vec. Master's thesis. University of New Orleans. https://scholarworks.uno.edu/td/1922.

[21] Sandra G. Hart and Lowell E. Staveland. 1988. Development of NASA-TLX (task load index): Results of empirical and theoretical research. In Human Mental Workload, Peter A. Hancock and Najmedin Meshkati (Eds.). Advances in Psychology, Vol. 52. North-Holland, 139-183. DOI : https://doi.org/10.1016/S0166-4115(08)62386-9

[22] Apple Inc. 2022. Change Zoom preferences for accessibility on Mac - Apple Support. Retrieved from https://support. apple.com/guide/mac-help/change-zoom-preferences-for-accessibility-mh40579/mac.

[23] Julie A. Jacko, Armando B. Barreto, Gottlieb J. Marmet, Josey Y. M. Chu, Holly S. Bautsch, Ingrid U. Scott, and Robert H. Rosa, Jr. 2000. Low vision: The role of visual acuity in the efficiency of cursor movement. In Proceedings of the 4th International ACM Conference on Assistive Technologies (Arlington, Virginia) (Assets'00). ACM, New York, NY, 1-8. DOI : https://doi.org/10.1145/354324.354327 
[24] Derry Jatnika, Moch Arif Bijaksana, and Arie Ardiyanti Suryani. 2019. Word2Vec model analysis for semantic similarities in English words. Procedia Comput. Sci. 157 (2019), 160-167. DOI : https://doi.org/10.1016/j.procs.2019.08.153

[25] Xiaolin Jin, Shuwu Zhang, and Jie Liu. 2018. Word semantic similarity calculation based on Word2vec. In Proceedings of the International Conference on Control, Automation and Information Sciences (ICCAIS). 12-16. DOI : https://doi.org/ 10.1109/ICCAIS.2018.8570612

[26] Tom Kenter and Maarten de Rijke. 2015. Short text similarity with word embeddings. In Proceedings of the 24th ACM International on Conference on Information and Knowledge Management (CIKM'15). Association for Computing Machinery, New York, NY, 1411-1420. DOI : https://doi.org/10.1145/2806416.2806475

[27] Rushil Khurana, Duncan McIsaac, Elliot Lockerman, and Jennifer Mankoff. 2018. Nonvisual interaction techniques at the keyboard surface. In Proceedings of the CHI Conference on Human Factors in Computing Systems (CHI'18). Association for Computing Machinery, New York, NY, 1-12. DOI : https://doi.org/10.1145/3173574.3173585

[28] Richard L. Kline and Ephraim P. Glinert. 1995. Improving GUI accessibility for people with low vision. In Proceedings of the SIGCHI Conference on Human Factors in Computing Systems (CHI'95). ACM Press/Addison-Wesley Publishing Co., New York, NY, 114-121. DOI : https://doi.org/10.1145/223904.223919

[29] Hae-Na Lee, Vikas Ashok, and I. V. Ramakrishnan. 2021. Bringing things closer: Enhancing low-vision interaction experience with office productivity applications. Proceed. ACM on Hum.-comput. Interact. 5, EICS (2021), 1-18.

[30] Hae-Na Lee, Sami Uddin, and Vikas Ashok. 2020. TableView: Enabling efficient access to web data records for screenmagnifier users. In Proceedings of the 22nd International ACM SIGACCESS Conference on Computers and Accessibility (ASSETS'20). Association for Computing Machinery, New York, NY. DOI : https://doi.org/10.1145/3373625.3417030

[31] V. Kathlene Leonard, Julie A. Jacko, and J. J. Pizzimenti. 2006. An investigation of handheld device use by older adults with age-related macular degeneration. Behav. Inf. Technol. 25, 4 (2006), 313-332.

[32] Longzhuang Li, Yonghuai Liu, Abel Obregon, and Matt Weatherston. 2007. Visual segmentation-based data record extraction from web documents. In Proceedings of the IEEE International Conference on Information Reuse and Integration. IEEE, 502-507.

[33] Wei Liu, Xiaofeng Meng, and Weiyi Meng. 2009. Vide: A vision-based approach for deep web data extraction. IEEE Trans. Knowl. Data Eng. 22, 3 (2009), 447-460.

[34] Valentyn Melnyk, Vikas Ashok, Yury Puzis, Andrii Soviak, Yevgen Borodin, and I. V. Ramakrishnan. 2014. Widget classification with applications to web accessibility. In Web Engineering, Sven Casteleyn, Gustavo Rossi, and Marco Winckler (Eds.). Springer International Publishing, Cham, 341-358.

[35] Gengxin Miao, Junichi Tatemura, Wang-Pin Hsiung, Arsany Sawires, and Louise E. Moser. 2009. Extracting data records from the web using tag path clustering. In Proceedings of the 18th International Conference on World Wide Web (WWW'09). Association for Computing Machinery, New York, NY, 981-990. DOI : https://doi.org/10.1145/1526709. 1526841

[36] Microsoft. 2021. Use Magnifier to make things on the screen easier to see. Retrieved from https://support.microsoft. com/en-us/help/11542/windows-use-magnifier-to-make-things-easier-to-see.

[37] Tomas Mikolov, Kai Chen, Greg Corrado, and Jeffrey Dean. 2013. Efficient estimation of word representations in vector space. arXiv e-prints, Article arXiv:1301.3781 (Jan. 2013).

[38] Lourdes Moreno, Xabier Valencia, J. Eduardo Pérez, and Myriam Arrue. 2018. Exploring the web navigation strategies of people with low vision. In Proceedings of the International Conference on Human Computer Interaction (Interacción'18). Association for Computing Machinery, New York, NY. DOI : https://doi.org/10.1145/3233824.3233845

[39] Afra Pascual, Mireia Ribera, Toni Granollers, and Jordi L. Coiduras. 2014. Impact of accessibility barriers on the mood of blind, low-vision and sighted users. Procedia Comput. Sci. 27 (2014), 431-440. DOI : https://doi.org/10.1016/j.procs. 2014.02.047

[40] Christopher Power, André Freire, Helen Petrie, and David Swallow. 2012. Guidelines are only half of the story: Accessibility problems encountered by blind users on the web. In Proceedings of the SIGCHI Conference on Human Factors in Computing Systems (CHI'12). ACM, New York, NY, 433-442. DOI : https://doi.org/10.1145/2207676.2207736

[41] Alisha Pradhan, Kanika Mehta, and Leah Findlater. 2018. "Accessibility came by accident": Use of voice-controlled intelligent personal assistants by people with disabilities. In Proceedings of the CHI Conference on Human Factors in Computing Systems (CHI'18). Association for Computing Machinery, New York, NY, 1-13. DOI: https://doi.org/10. 1145/3173574.3174033

[42] Jyotika Prasad and Andreas Paepcke. 2008. CoreEx: Content extraction from online news articles. In Proceedings of the 17th ACM Conference on Information and Knowledge Management (CIKM'08). Association for Computing Machinery, New York, NY, 1391-1392. DOI : https://doi.org/10.1145/1458082.1458295

[43] Radim Ǩehůřek, Petr Sojka, et al. 2011. Gensim-statistical semantics in Python. Retrieved from Genism.org.

[44] Johnny Saldaña. 2021. The Coding Manual for Qualitative Researchers. Sage.

[45] Freedom Scientific. 2022. ZoomText Screen Magnifier and Screen Reader - zoomtext.com. Retrieved from https://www. zoomtext.com/. 
[46] Chengyao Shen and Qi Zhao. 2014. Webpage saliency. In Computer Vision - ECCV 2014, David Fleet, Tomas Pajdla, Bernt Schiele, and Tinne Tuytelaars (Eds.). Springer International Publishing, Cham, 33-46.

[47] Dimitris Spiliotopoulos, Gerasimos Xydas, Georgios Kouroupetroglou, Vasilios Argyropoulos, and Kalliopi Ikospentaki. 2010. Auditory universal accessibility of data tables using naturally derived prosody specification. Univers. Access Inf. Soc. 9, 2 (June 2010), 169-183. DOI : https://doi.org/10.1007/s10209-009-0165-0

[48] Sarit Felicia Anais Szpiro, Shafeka Hashash, Yuhang Zhao, and Shiri Azenkot. 2016. How people with low vision access computing devices: Understanding challenges and opportunities. In Proceedings of the 18th International ACM SIGACCESS Conference on Computers and Accessibility (ASSETS'16). ACM, New York, NY, 171-180. DOI: https://doi. org/10.1145/2982142.2982168

[49] Mary Theofanos and Ginny Redish. 2005. Helping low-vision and other users with Web sites that meet their needs: Is one site for all feasible? Technic. Commun. 52 (2005), 9-20.

[50] E. Ukkonen. 1995. On-line construction of suffix trees. Algorithmica 14, 3 (Sept. 1995), 249-260. DOI : https://doi.org/ 10.1007/BF01206331

[51] Yan Wen, Qingtian Zeng, Hua Duan, Feng Zhang, and Xin Chen. 2018. An automatic web data extraction approach based on path index trees. Int. F. Performab. Eng. 14, 10 (2018). DOI : https://doi.org/10.23940/ijpe.18.10.p21.24492460

[52] Kristin Williams, Taylor Clarke, Steve Gardiner, John Zimmerman, and Anthony Tomasic. 2019. Find and seek: Assessing the impact of table navigation on information look-up with a screen reader. ACM Trans. Access. Comput. 12, 3 (Aug. 2019). DOI : https://doi.org/10.1145/3342282

[53] Yanhong Zhai and Bing Liu. 2005. Web data extraction based on partial tree alignment. In Proceedings of the 14th International Conference on World Wide Web (WWW'05). Association for Computing Machinery, New York, NY, 7685. DOI : https://doi.org/10.1145/1060745.1060761

[54] Quanlong Zheng, Jianbo Jiao, Ying Cao, and Rynson W. H. Lau. 2018. Task-driven webpage saliency. In Computer Vision - ECCV 2018, Vittorio Ferrari, Martial Hebert, Cristian Sminchisescu, and Yair Weiss (Eds.). Springer International Publishing, Cham, 300-316.

[55] Jun Zhu, Zaiqing Nie, Ji-Rong Wen, Bo Zhang, and Wei-Ying Ma. 2006. Simultaneous record detection and attribute labeling in web data extraction. In Proceedings of the 12th ACM SIGKDD International Conference on Knowledge Discovery and Data Mining (KDD’06). Association for Computing Machinery, New York, NY, 494-503. DOI : https://doi.org/10.1145/1150402.1150457

Received June 2021; revised February 2022; accepted February 2022 